\title{
Metallized SU-8 thin film patterns on stretchable PDMS
}

Tiffany Baëtens, Emiliano Pallecchi, Vincent Thomy, and Steve Arscott

Institut d'Electronique, de Microélectronique et de Nanotechnologie (IEMN), CNRS UMR8520, The University of Lille, Cité Scientifique, 59652 Villeneuve d'Ascq, France.

\section{Abstract}

We show how a rigid patterned SU-8 thin film in tandem with the mechanical Poisson effect can be used beneficially to enable robust metallization on stretchable polydimethylsiloxane (PDMS). Two generic planar processes used to form a thin, mechanically-robust strain-shielding photoresist on a soft substrate are developed to demonstrate these ideas. The first process is a self-aligned metallization of photolithographically patterned SU-8 features on PDMS. The second process is a fully photolithographic lift-off metallization of pre-patterned SU-8 features on PDMS. In both cases, the SU-8 has a sub-micrometre thickness ( $800 \mathrm{~nm}$ ) - supporting a thermally evaporated $\mathrm{Ti} / \mathrm{Au}(5 \mathrm{~nm} / 50 \mathrm{~nm})$ thin film metallization. The resulting samples were characterized electromechanically-the results demonstrate that the electrical continuity of metal lines (width $=150 \mu \mathrm{m}$ and length up to $1 \mathrm{~cm}$ ) is maintained up to $\sim 70 \%$ strain between the lines. The electrical resistance of such lines remains relatively stable: $35 \Omega$ at zero-strain and $63 \Omega$ at $69 \%$ average interline strain. The electrical resistivity of the evaporated gold is near to that of pure gold, and remains so even at high strains ( $49 \mathrm{n} \Omega \mathrm{m}$ at $10 \%$ and $70 \mathrm{n} \Omega \mathrm{m}$ at $41 \%$ )this proves the absence strain-induced micro-cracking of the metal lines. Although a specific 
evaporated metal combination is used here to demonstrate a working prototype system, in principle, any thin-film material, which can be deposited by microfabrication, e.g. insulators, semiconductors..., could be integrated into the generic processes. The basic lithographic processes expounded here are also potentially extendible to encompass the integration of other microfabrication techniques, e.g. soft lithography, contact printing..., enabling more complex flexible systems to be envisaged and realized.

Keywords: Stretchable electronics, photolithography, planar processes, metallization, PDMS, SU-8, Poisson effect 


\section{Introduction}

The means of fabricating robust flexible, stretchable, and squashable systems is evolving rapidly due to the demands of a multitude of emerging applications, e.g. electronic skin [1], patch technologies [2], nanoelectronics [3], integrated circuits [4], flexible displays [5], optics [6], communications [7], energy storage [8], smart clothes [9], bioMEMS [10], smart neural interfaces [11] and soft robotics [12]. Such systems aim to combine components (pads, tracks, and devices) on a soft substrate to form mechanically-robust systems. Flexible and in particular stretchable/squashable technologies and applications [13-15] require that the systems' components are mechanically and electrically robust at high strain values $>10 \%$. There are two main approaches for the manufacture of such systems. First, fabrication on a host wafer and subsequent transfer to a flexible or stretchable polymeric substrate. Second, a direct fabrication onto the polymeric substrate. An example of the former is 'transfer printing' which has proved to be powerful-however such approaches can suffer from scalability issues at sub-micrometre dimensions, alignment problems, and 3D limitations [16]. Alternatively, direct fabrication onto a polymeric substrate would be very straightforward-eliminating the need for a transfer step. As such, it does not require the optimization of adhesion difference between transfer and host substrate that is mandatory for transfer printing. Therefore, direct fabrication is faster to develop and less dependent on the specific properties of the substrate. However, this approach brings practical difficulties which need to be overcome. The reason for this is the evident incompatibility of some physical properties of soft materials with the standard planar approach, e.g. the large thermal expansion coefficient and unfavourable surface wetting properties of elastomers such as polydimethylsiloxane (PDMS). For example, unless metallic features are 
small [17], metallizing thin film based tracks directly onto PDMS invariably results in film cracking (both microcracking [18] and macroscopic cracking [19]) leading to a rapid rise in the electrical resistance and resistivity with strain [18] and possible system failure-although it should be noted that cracking can sometimes be beneficial [20]. In order to overcome these problems, some hybrid solutions have been demonstrated in the literature. These approaches usually involve shielding key system elements from the strain, e.g. placing elements at a neutral axis [21] in the case of a flexible system, or by inserting a rigid element [22-24] in proximity to the device to be shielded. Some solutions involve enabling free-standing elements [25]. However, often such solutions are not as compatible with simple planar fabrication processes as the process engineer would wish.

Here we demonstrate how very thin SU-8 patterns can form a mechanically robust patterned thin film on the surface of PDMS. This SU-8 patterns can be subsequently metallized using standard photolithographic techniques. The resulting metal lines are more electromechanically robust than lines directly metallized onto PDMS [20], the high strain electrical resistance differing little from the zero-strain resistance-and the high strain resistivity of the thin film gold approaching that of pure gold.

\section{Microfabrication}

2.1. Choice of materials and their relevant properties 
The photoresist SU-8 [26] was chosen to form the mechanically-robust thin film on the PDMS membranes. First, SU-8 can be easily photolithographically patterned. Second, thin submicrometre films can easily be spin coated onto quasi-flat surfaces using low viscosity SU-8 mixtures-enabling the forming of thin, robust planar features. Third, our initial process optimization found that its adhesion to PDMS is good if the PDMS surface is treated with an appropriate oxygen plasma [19] prior to spin-coating of the SU-8. Fourth, SU-8 is compatible with many applications [27] - being both chemically robust and biocompatible for the everincreasing number of life science applications. Finally, the mechanical properties of the polymerized SU-8 [28,29] are such that sub-micrometre patterned thin films will be mechanically robust-in this way, the metallization on top of the SU-8 will be less prone to cracking upon straining [30]. Indeed, a digital image correlation study of metallized SU-8 showed the absence of microcracking in an evaporated nanometre thick thin film [30]. There has already been efforts combining SU-8 and PDMS in various innovative processes [31,32], e.g. large SU-8 features directly on PDMS [33]. First, it is well-documented that SU-8 can be used to create master moulds for the manufacture of shaped PDMS parts [34]. Second, in terms of embedding the polymerized SU-8 in a final system, this can be achieved by using an integrated approach of separated materials [35-37] or a hybrid approach by combining them in a mixture prior to curing to provide an SU-8/PDMS material with tuneable physical properties depending on the specific mixing proportions [38].

The mechanical properties of the materials are very important in the context of the fabrication of flexible and stretchable electronics and microsystems. Polymerized SU-8 has mechanical properties which depend on processing conditions [28]. The elastic (Young's) 
modulus of SU-8 is in the range 1.7-2.2 GPa [28]. The ultimate tensile strength (uts) of SU-8 is reported to be in the range 34-130 MPa $[29,39,40]$. PDMS also has mechanical properties which depend strongly on processing conditions. For the PDMS processing here, the values of $E$, uts, and ultimate tensile strain are 2.6 $\mathrm{MPa}, 5.3 \mathrm{MPa}$, and 75\% respectively [41]. Evaporated gold has an uts of tens to hundreds of MPa depending on evaporation conditions [42]. Evaporated titanium has an ultimate tensile strength of the order of $300 \mathrm{MPa}$-also depending on evaporation conditions. A Titanium/Gold (Ti/Au) bilayer metallization-having a thickness of 5 $\mathrm{nm} / 50 \mathrm{~nm}$ - was deliberately chosen as this combination is known to crack at low strains when deposited directly onto PDMS [20]. By using this metallization, any contribution of the SU-8 will be apparent in the measurements. In addition, evaporated Ti has a higher bond strength to SU-8 than evaporated gold [43]. In terms of the electrical measurements, evaporated gold filmshaving a thickness of $\sim 60 \mathrm{~nm}$-have been measured to have a room temperature electrical resistivity $\rho$ of $\sim 30 \mathrm{n} \Omega \mathrm{m}[44]$. 


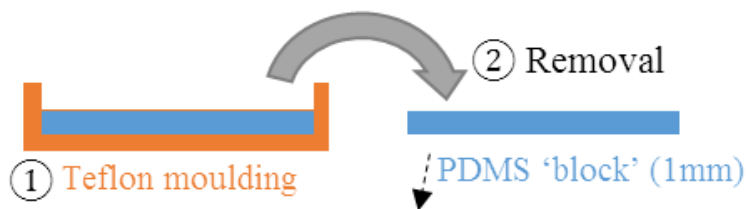

(3) $\mathrm{O}_{2}$ plasma $1700 \mathrm{~J}-0.4 \mathrm{mbar}$

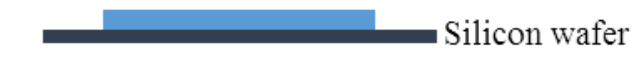

Spin coat

(4) SU8 $2002(800 \mathrm{~nm})$

(4) $2000 / 500 / 30 \mathrm{~s}$
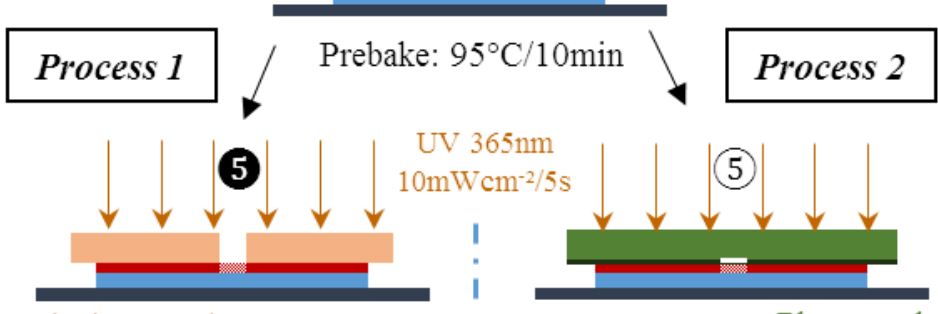

Shadow mask

(6) Postbake: $95^{\circ} \mathrm{C} / 10 \mathrm{~min}$

Photomask

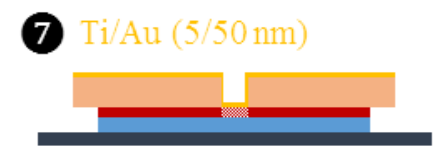

8 Remove shadow mask
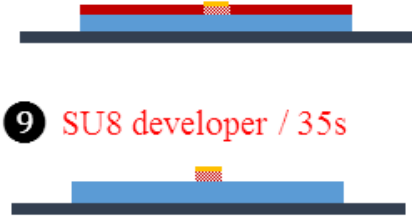

10 IPA rince and $\mathrm{N}_{2}$ dry

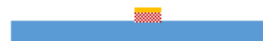

(7) SU8 developer $/ 35 \mathrm{~s}$

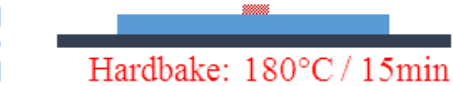

(8)

AZ1518

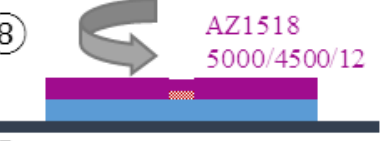

(9) Bake: $95^{\circ} \mathrm{C} / 120 \mathrm{~s}$

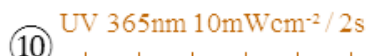

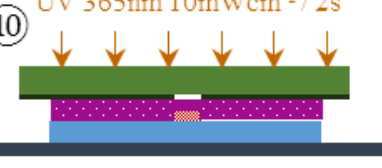

(11) Develop: MIF326/20s

(12) $\mathrm{Ti} / \mathrm{Au}(5 / 50 \mathrm{~mm})$

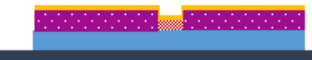

(13) Lift-off Remover PG

Remove PDMS from silicon wafer

PDMS

Photomask

Metallization

Silicon wafer

Shadow mask

SU8 2002 (exposed)

SU8 2002

AZ 1518

AZ 1518 (non-exposed) 
Figure 1. The two microfabrication processes developed in the study for the fabrication of the mechanically robust metallized SU-8 patterned thin film on PDMS. An auto-aligned process (Process 1) using a physical shadow mask and pre-moulded PDMS-left hand image, a fully photolithographic lift-off process (Process 2) using a photomask and pre-moulded PDMS-right hand image. The photomask (green) is composed of a thick quartz $(1 \mathrm{~mm})$ block patterned with thin film chromium layer.

\subsection{Metallized SU-8 thin films on PDMS}

Two original microtechnological processes were developed for the fabrication of the samplessee Figure 1. The first process (Process 1) involves metallization using a physical shadow mask and a thick ( 1 $\mathrm{mm}$ ) pre-fabricated block of PDMS. This is an auto-aligned process as the shadow mask is not removed until after the metallization of the sample-see left-hand process in Figure 1. The second process (Process 2) involves lift-off metallization via the use of a photoresist mask - this process is a fully photolithographic process (see right image in Figure 1) also involves a thick ( $1 \mathrm{~mm})$ pre-fabricated block of PDMS. The two processes share the same root up until the first photolithographic step of Process 2-this initial joint process is carried out as follows. The two components of a PDMS kit (Sylgard 184-Dow Corning, USA) are mixed to a have a 10:1 volume composition-giving known mechanical properties [41]. This mixture is poured into round, flat-bottomed Teflon dishes ( $\sim 3$-inch diameter) and outgassed in a rotary pump-driven vacuum chamber $\left(\sim 10^{-4}\right.$ mbar for 30 minutes). The volume of the mixture is calculated to obtain a PDMS thickness of $1 \mathrm{~mm}$. The filled dishes are placed on a level hotplate (initially at a temperature of $20^{\circ} \mathrm{C}$ ) for 10 minutes to ensure a uniformly thick PDMS layer. The 
hotplate is subsequently ramped to $80^{\circ} \mathrm{C}\left(\sim 1^{\circ} \mathrm{C} \mathrm{s}^{-1}\right)$ and the samples are pre-cured for $1 \mathrm{~h}$. Finally, the dishes are placed in a box furnace at $100^{\circ} \mathrm{C}$ for 30 minutes-a cure temperature/time advised by the commercial supplier. The round PDMS layers are extracted from the Teflon dishes and cut-using a scalpel-to form rectangular samples having lateral dimensions measuring $\sim 1.5 \times 2 \mathrm{~cm}$. During cutting, avoidance with sources of fibres and particles, e.g. cut cleanroom paper, is important to maintain clean, particle-free PDMS surfaces essential for the spin-coating of the SU-8-we observe that PDMS is prone to being a 'getter' of such particulate contaminants. The thickness of the PDMS samples was measured to be $980 \pm 5 \mu \mathrm{m}$. The PDMS samples are then placed onto a 3-inch diameter silicon wafer-the PDMS edge being orientated to the $\langle 110\rangle$ silicon wafer flat in order to be able to cleave the wafer to the same size as the PDMS sample later in the process. At this point, a nitrogen gun aides the adhesion of the PDMS to the silicon surface-other methods, e.g. pressing with object such as tweezers, should be avoided as we observed that this enhanced dewetting of the SU-8 resist during the subsequent spin coating of the SU-8. The PDMS surfaces are then treated to an optimized oxygen plasma [19] $\left(\right.$ Energy dose $=1700 \mathrm{~J}-\mathrm{O}_{2}$ pressure $=0.5 \mathrm{mbar}$ ) - it was observed that this step strongly enhances wetting of the SU-8 photoresist to the PDMS during the subsequent spin-coating. It was also observed that the specific does is critical $-<1500 \mathrm{~J}$ leads to reduced adhesion, $>1800 \mathrm{~J}$ leads to cracking on the surface of the PDMS. The PDMS surfaces are spin coated with SU-8 2002 (Microchem, USA). A uniform 800 nm thick SU-8 film is achieved by spin coating at 2000 rpm for 30s followed by a pre-bake (in a box oven) at $95^{\circ} \mathrm{C}$ for 10 minutes. It should be noted that a relatively good adhesion between the PDMS sample and the silicon wafer ensures that the PDMS remains in contact with the silicon wafer-note that delamination of the PDMS from 
the silicon during the process results in wrinkle formation due to thermal expansion (see Supplementary Information Figure S2).

The result is a patterned SU-8 thin film on the PDMS that are topographically smooth. This is easily explained as the relatively low linear thermal expansion coefficient of crystalline silicon $\left(\alpha_{S i}=2.6 \times 10^{-6}{ }^{\circ} \mathrm{C}^{-1}\right)$ compared to that of PDMS $\left(\alpha_{P D M S}=9.6 \times 10^{-4}{ }^{\circ} \mathrm{C}^{-1}\right)$ which prevents the PDMS from expanding on curing (pre-baking and post-baking) of the SU-8. It should also be noted that an occasional minor dewetting $(<500 \mu \mathrm{m}$ wide) of the SU- 8 film is observed at the PDMS sample edges-more work is needed to optimize this, although it is observed that making sure the SU-8 is in contact with the PDMS sample edges improves this problem. To end the common phase of the two processes, the silicon wafer is cleaved to have a similar size to the PDMS sample. Following this, the two processes take different routes.

\subsection{Lithography and metallization of the SU-8 thin films}

For Process 1 (self-aligned metallization), a physical shadow mask-composed of a 50 um thick Ni metal film containing the pre-etched metallization patterns (see Supplementary Information Figure S1 for different types of masks used)-is placed onto the surface of the SU8/PDMS/silicon. The sample is then fixed onto a commercial evaporation chuck using sprung clips to hold the physical mask/SU-8/PDMS/silicon ensemble firmly in place. The pre-baked SU8-which is visible through the opening in the shadow mask-is then photolithographically exposed [45] to UV light $(365 \mathrm{~nm})$ for 5 seconds at $10 \mathrm{~mW} \mathrm{~cm}^{-2}$ in a commercial mask aligner 
(Suss Microtech, Germany). The ensemble is then placed into a box oven at $95^{\circ} \mathrm{C}$ for 10 minutes in order to polymerize the UV-exposed SU-8 photoresist. At this point it should be noted that in addition to the PDMS being adhered to the silicon, the mechanical mask fixing also avoids delamination and thermal expansion of the PDMS. The ensemble is metallized $(5 \mathrm{~nm} / 50 \mathrm{~nm}$ $\mathrm{Ti} / \mathrm{Au}$ ) in a commercial MEB $550 \mathrm{~S}$ electron beam evaporation system (Plassys, France). The shadow mask is then removed and the photolithographically unexposed, non-polymerized, nonmetallized SU-8 is then removed from the surface of the PDMS by plunging the sample in a commercial SU-8 developer solution (1-Methoxy-2-propyl acetate) for $35 \mathrm{~s}$. Finally, the sample is rinsed with isopropanol (IPA) and dried with nitrogen gas.

For Process 2 (fully photolithographic), the SU-8/PDMS/Silicon ensemble is placed into the commercial mask aligner. The SU-8 patterns are exposed to UV ( $365 \mathrm{~nm}-2 \mathrm{~s} / 10 \mathrm{~mW} \mathrm{~cm}{ }^{-2}$ ) via a commercial photomask in soft contact mode to avoid potential problems which could arise due to direct contact of the photomask and the sample surface. Interestingly, it was observed that a better resolution was apparent in narrower features - this is likely to be due to UV reflection from silicon host [45]. Despite being in soft contact mode to enhance resolution, we anticipated that slight sample crushing that could possibly damage the SU-8 film via the Poisson effect-but this was not apparent. The sample is then post-exposure baked in a box oven at $95^{\circ} \mathrm{C}$ for 10 minutes to polymerize the SU-8. The unexposed SU-8 is then removed from the surface of the PDMS by exposure to a commercial SU-8 developer solution (1-Methoxy-2-propyl acetate) for $35 \mathrm{~s}$. This latter step needs to be done before the later thermal evaporation step as it has been observed that SU-8 can be chemical modified during metallization-probably due to UV emission from the hot metallization target. A hardbake step $\left(180^{\circ} \mathrm{C}\right.$ for $\left.20 \mathrm{~min}\right)$ can now be 
performed which cannot be done using Process 1 due to presence of photolithographically unexposed SU-8. The goal of this step is twofold: (i) improved adhesion between the SU-8 and the PDMS, and (ii) improved chemical inertness of the SU-8 films. Following the hardbake, the sample is then spin coated with a positive photoresist (AZ1518) to have a thickness of $\sim 1.2 \mu \mathrm{m}$. The AZ1518 is pre-baked in an oven at $95^{\circ} \mathrm{C}$ for 2 minutes. In an effort to form a lift-off overhang feature, the unexposed AZ1518 is dipped in AZ326 developer for 30s. After drying, the sample is again loaded into the commercial mask aligner. The photomask is aligned to the now visible SU8 thin film patterns - and the AZ1518 is exposed ( $365 \mathrm{~nm}-2 \mathrm{~s}$ and $10 \mathrm{~mW} \mathrm{~cm} \mathrm{~m}^{-2}$ ). The exposed AZ1518 is then removed from the sample surface using a commercial developer solution MIF726 for 20-25 s. Some film cracking was observed at this point originating from sharp corners but this did not cause problems later in the process. The patterned AZ1518/SU8/PDMS/silicon ensemble then is metallized $(5 \mathrm{~nm} / 50 \mathrm{~nm} \mathrm{Ti} / \mathrm{Au}$ ) in the commercial electron beam evaporation system. Finally, the AZ1518 is removed using a commercial positive photoresist remover (Remover PG at $70^{\circ} \mathrm{C}$ ) - leading to the lift-off of the Ti/Au metallization where it is not required.

\section{Experimental results}

\subsection{Topographic features resulting from the processes}

By using the two different processes in combination with the different shadow masks and photomasks several samples were fabricated allowing mechanical and electromechanical 
testing of $\sim 70$ lines. The observed topographies of metallized SU-8 thin film patterns on PDMS are shown in Figure 2.

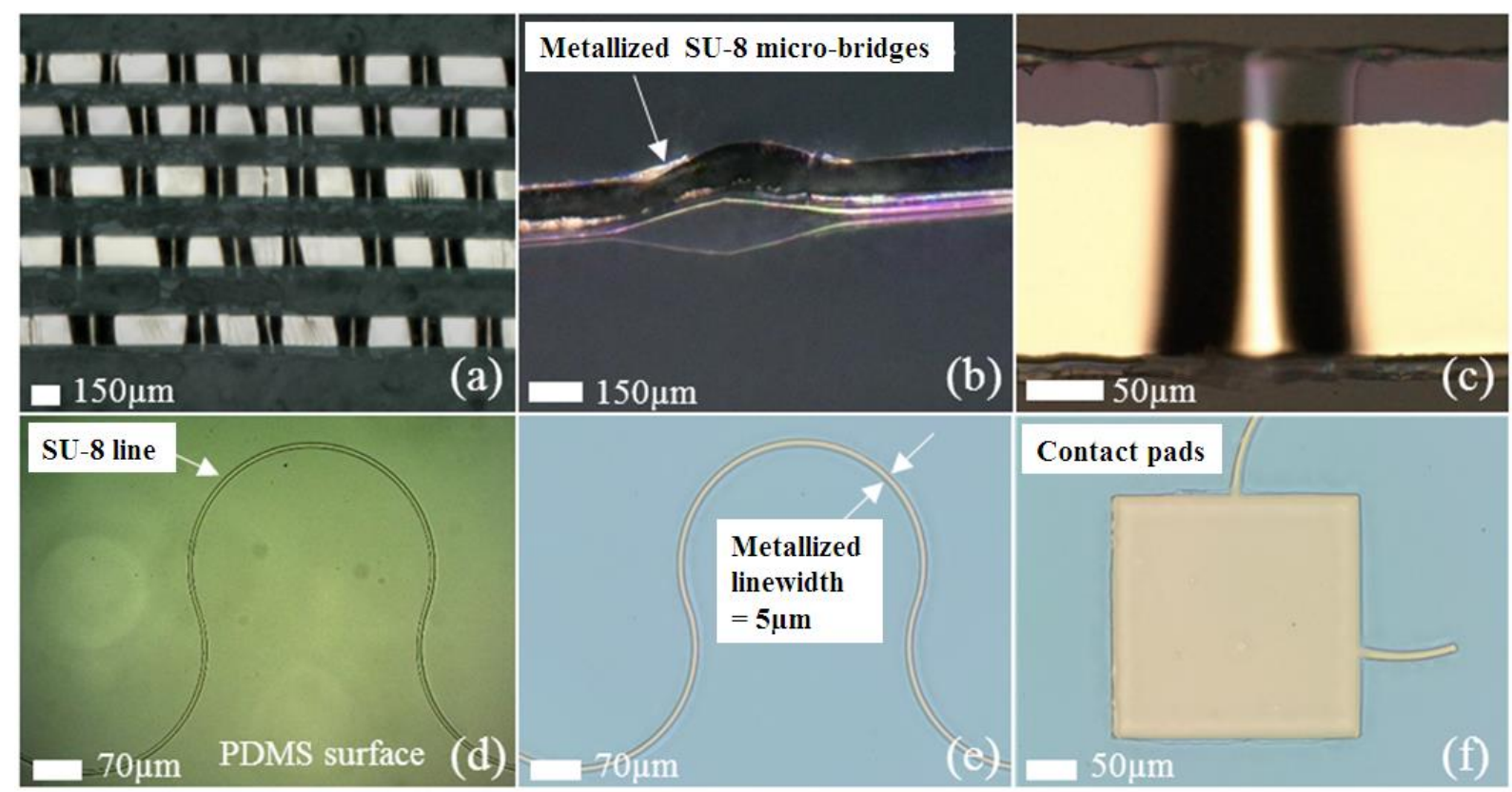

Figure 2. Different topographies of metallizations/SU-8 thin films on PDMS obtained using the two microfabrication processes. (a-c) Lines containing micro-bridges obtained with Process 1. (d-f) topographically flat lines obtained using Process 2. A delaminated micro-bridge is clearly seen in (b) whereas the lithographic resolution of $\sim 5 \mu \mathrm{m}$ is clearly seen in SU-8 lines patterned onto PDMS (d) which can be subsequently lithographically metallized (e).

Depending on which process is used and certain key steps in the process, three main topographies of metallized SU-8 thin film patterns on PDMS were observed. First, long lines containing periodically-spaced micro-bridge structures were observed using Process 1-see Fig. 2(a). The micro-bridges themselves are composed of the metallized SU-8 film which has locally 
detached from the PDMS surface; indicating a combination of mechanical process stress and low film adhesion-see Fig. 2(b) and Fig. 2(c). We observe that the height ( $29 \mu \mathrm{m})$ and length $(\sim 130 \mu \mathrm{m})$ of the micro-bridges as well as the distance between the micro-bridges $(\sim 550 \mu \mathrm{m})$ is relatively large compared to film thicknesses. 'As the micro-bridges contribute to the flexibility of the structure, it is important that their reproducibility (dimensions and spacing) is high. The high reproducibility of our process was verified by comparing the micro-bridges obtained on three separate samples. Second, very narrow ( $2 \mu \mathrm{m}$ width) topographically flat SU-8 lines can be obtained using Process 2-see Fig. 2(d). Using the lift-off step, these lines can be metallized and remain topographically flat-see Fig. 2(e). Topographically flat pads having a large area can also be obtained using Process 2-see Fig. 2(f). In addition to these findings, we also observed that the wrinkled lines can be created if the PDMS block inadvertently detached from the silicon wafer during the initial SU-8 pre-baking step. Wrinkling can occur in narrow lines and large surfaces (see Supplementary Information Section 2). The wrinkles have a period $\sim 50 \mu \mathrm{m}$ which can be predicted using modelling based on thermal expansion and mechanical properties-see Supplementary Information. The horseshoe shaped lines shown in Fig. 2 were tested mechanically with their longest length orientated transversally to the longitudinal direction of the applied strain. Cracking appeared is all lines tested beyond $\sim 5 \%$ average interline strainsee Supplementary Figure S4. This is due to a large component of the length of the line in the direction of the applied strain.

The micro-bridges obtained by Process 1 occur between step 9 and 10 (refer to Figure 1). When the unexposed SU-8 is developed and the PDMS is removed from the silicon wafer, residual process-induced stress relaxes that can lead to the formation of micro-bridges in 
metallized SU-8 thin film. In an effort to explain this, it is thought that the absence of the $180^{\circ} \mathrm{C}$ SU-8 hardbake step in Process 1 leads to a lower adhesion between the SU-8 and the PDMSthus any stress relaxation causes delamination. Interestingly, it was observed that film wrinkling can sometimes occur at step 7 (refer to Figure 1) during Process 2. In both cases, it is thought that the mechanical stress generated by PDMS-caused by thermal expansion: either detachment of on the surface-plays a role in governing the resulting topography. We also note that there will be a contribution from the intrinsic stresses caused by e.g. the polymerization of the SU-8 [46], although this is likely to be low compared to those caused by any thermal expansion of the PDMS. Finally, it should be noted that the fabricated lines (Ti/Au on SU-8) contain no process-induced cracks. This can be compared to PDMS directly metallized using Ti/Au where process-induced cracking is present [20].

\subsection{Mechanical straining of the samples}

First, it is important to make the distinction between the 'applied strain' which we apply using the setup, the 'interline strain' measured between adjacent lines in the sample during the study, and the 'average interline strain' which is the average of all the measured 'interlines strains' at a given 'applied strain' value. Using our in-house strain applicator, we impose the applied strain on our samples under test. The interline strain is calculated directly from imaging our samples at different points using the microscope at each stretching following equation: $\varepsilon_{l o c}=\Delta L_{l o c} / L_{l o c}$, where $\varepsilon_{l o c}$ is the calculated interline strain, $L_{l o c}$ is the length between two

distinct points (e.g. corners of lines) at the beginning of the experiment, and $\Delta L_{l o c}$ represents 
the change of the length between the two points. These lengths are measured as components in the longitudinal and transverse directions with the respect of the sample edges. The lengths were carefully measured using a digital microscope VHX-6000 (Keyence, Japan). For many applications what interest us the most is interline strain in PDMS, i.e. the interline strain. By averaging the interline strains from certain points on the sample surface, the average interline strain $\varepsilon_{\text {ave }}$ can be used to plot the measurements. As will be seen, the average interline strain depends of the overall architecture of the sample-this should be taken into consideration in the design of flexible systems [17].

\subsubsection{Transversally-orientated Au/Ti/SU-8 lines on PDMS}

Figure 3 shows the measured electrical resistance $R$ and the electrical resistivity $\rho$ plotted as function of the average interline strain $\varepsilon_{a v e}$ for several metallized lines fabricated using two shadow masks. The electrical resistance of the lines was measured using a 2612B dual-channel system sourcemeter (SMU) running 'LabTracer 2.0' software (Keithley, USA), and a probe station using in-house sprung probes which were designed not damage the metallized SU-8 lines. Indeed, as an aside it was found that the metallized SU-8 on PDMS is much more resilient to multiple probing compared to metallized lines directly patterned onto PDMS [20] - even using standard non-sprung probes. The interline strain was measured from images of the sample under straining taken using an optical microscope using several fixed points, e.g. corners, of the metallized lines. The average interline strain used to plot Figure 3 is an averaging of these measurements. 
The resistivity $\rho$ of the lines was calculated using the following equation: $\rho=R w t / l$, where $R$ is the measured resistance of the lines, $w$ the width of the line, $t$ the thickness of gold of the line and $l$ the length between the probes for electrical testing. The dimensions of the lines were carefully measured at each applied straining using the digital microscope.
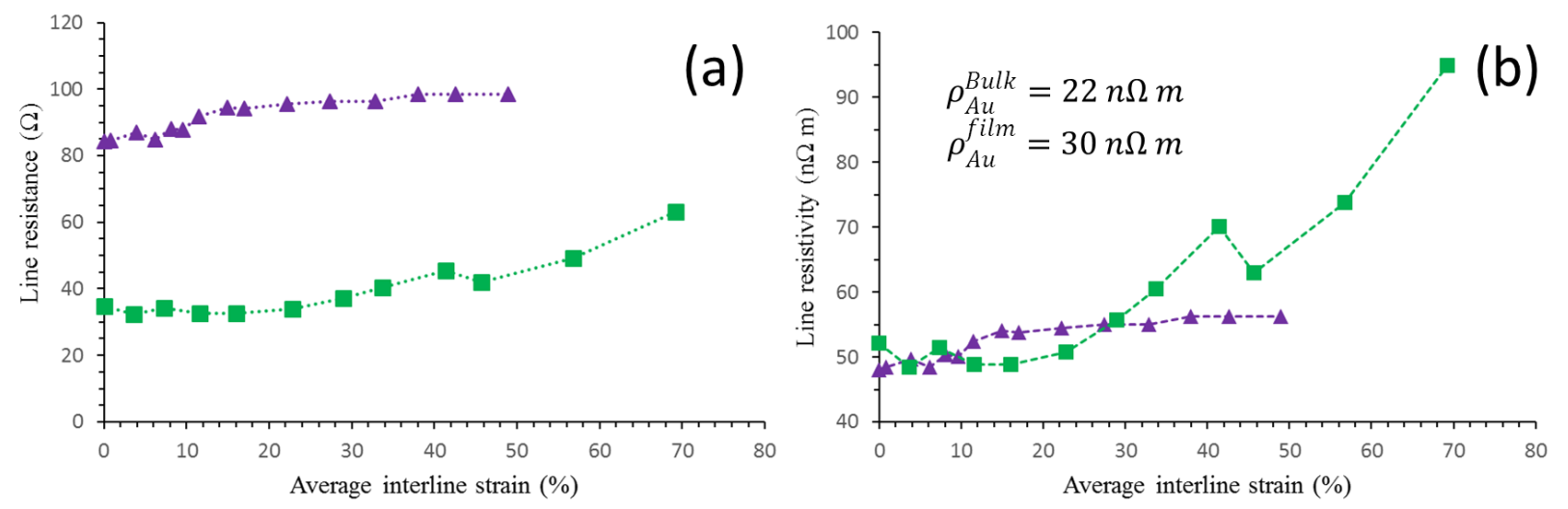

Figure 3. The measured electrical resistance (a) and resistivity (b) of Au/Ti/SU-8 transversallyorientated lines on PDMS as a function of the average interline strain. The results for two different masks are shown. Data points connected with unbroken lines shows resistance of the lines, whereas the data points connected with dashed lines represent the resistivity. The filled triangular data points $(\mathbf{\Delta})$ represent a line of shadow mask 1, the zero-strain dimensions of the line are $80 \mu \mathrm{m}$ (width) by $1 \mathrm{~cm}$ (length). The filled square data points ( $\mathbf{\square}$ ) represent an average measurement of $9 \mathrm{Au} / \mathrm{Ti} / \mathrm{SU}-8$ lines using mask 2, the zero-strain dimensions are $150 \mu \mathrm{m}$ (width) by $7.95 \mathrm{~mm}$ (length). Thickness of Au/Ti/SU-8 lines are 50/5/800 $\mathrm{nm}$. 
The first observation we can make is that the resistance of the lines remains relatively constant up to average interline stain value of $\sim 50 \%$. No microcracking or macroscopic cracking is observed in the $\mathrm{Au} / \mathrm{Ti} / \mathrm{SU}-8$ lines as is apparent in metallized PDMS lines whose resistance can increases dramatically with applied strain [47]. By using the above equation for the resistivity and taking care to account for any mechanically-induced changes in metallization dimension it is possible to extract the values of resistivity-which will evidently be dominated by the gold. These values of resistivity are also plotted on Fig. 3. In the literature, the room temperature resistivity thermally evaporated gold is typically around $30 \mathrm{n} \Omega \mathrm{m}$ for a $\sim 60 \mathrm{~nm}$ thick film [44]this can be compared to the resistivity of pure gold $\sim 22 \mathrm{n} \Omega \mathrm{m}\left(\sim 45 \times 10^{6} \mathrm{~S} \mathrm{~m}^{-1}\right)$. The average measured resistivity of $\mathrm{Au} / \mathrm{Ti} / \mathrm{SU}-8$ lines up to $30 \%$ average interline strain here is $51.2 \mathrm{n} \Omega \mathrm{m}$ $\pm 2.6 \mathrm{n} \Omega \mathrm{m}\left(\sim 19.5 \times 10^{6} \mathrm{~S} \mathrm{~m}^{-1}\right)$; and up to $50 \%$ average interline stain is $53.5 \pm 5.2 \mathrm{n} \Omega \mathrm{m}\left(\sim 18.7 \times 10^{6}\right.$ $\left.\mathrm{S} \mathrm{m}^{-1}\right)$. The applied strain was increased beyond $50 \%$ for the metallized SU-8 lines created using mask 2. It was observed that the resistance began to increase when the average interline strain was greater than $60 \%$-this was due to cracking.

\subsubsection{Straining of large area Au/Ti/SU-8 pads}

In addition to the metallized SU-8 lines, pads with different dimensions were tested. Large surface pads were tested as they are often a required part of an electronic system, either for external contacts of testing. Table 1 presents the dimensions of pads and the average inter-pad strain when the pads cracked. 
Pad dimensions $(\mu \mathrm{m})$

Measured inter-pad strain

(\%)

\begin{tabular}{|c|c|}
\hline $2000 \times 2000$ & $26.0 \pm 7.6$ \\
\hline $1000 \times 1000$ & $30.2 \pm 8.0$ \\
\hline $600 \times 600$ & $41.9 \pm 7.3$ \\
\hline $300 \times 300$ & $58.6 \pm 3.1$ \\
\hline $100 \times 100$ & No cracking observed \\
\hline
\end{tabular}

Table 1. Cracking of large area pads composed of Au/Ti/SU-8 lithographically patterned onto PDMS.

\subsubsection{Effect of line orientation on the straining of $A u / T i / S U-8$ lines on PDMS}

Several samples were fabricated in order to observe the mechanical and the electromechanical behaviour of metallized SU-8 line having different line orientation. Figure 4(a) shows the architecture of the sample. The sample contains $10 \mathrm{Au} / \mathrm{Ti} / \mathrm{SU}-8$ lines lithographically patterned onto a PDMS support. The orientation angle $\theta$ was varied from $0^{\circ}$ (traversal lines) to $90^{\circ}$ (longitudinal lines) in steps of $10^{\circ}$. The sample was mounted in the in-house strain applicator so as to enable longitudinal straining in the direction of the arrows shown in Fig. 4(c). The sample was strained and imaged using a digital microscope which enables a large, high resolution photograph to be assembled and subsequently analysed. The in-house strain applicator enables the applied strain to be increased in steps e.g. 5\%, 10\%. However, as it is known that the specific sample layout architecture plays a key role in determining the interline strain 19 points were 
chosen and measured on the sample at each applied strain. These values make up a rudimentary 'strain mapping' of the sample allowing the interline strain to be known and an average interline strain to be evaluated to enable plotting of the data.

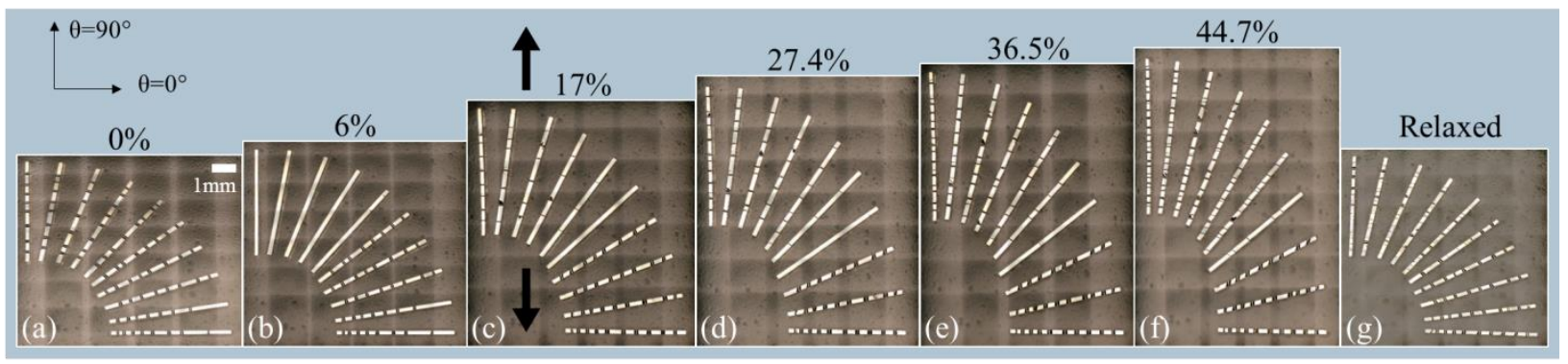

Figure 4. Optical microscopy images showing angled lines at different values of average interline strain (indicated on each pictures). The sample contains 10 lines with average interline strain varying from $0 \%(\mathrm{a})$ to $44.7 \%$ (f) and relaxed-sample at $0 \%$ applied strain after stretching (g). Straining is applied longitudinally to the sample indicated by black arrows in (c). All lines are $\mathrm{Au} / \mathrm{Ti} / \mathrm{SU}-8(5 \mathrm{~nm} / 50 \mathrm{~nm} / 800 \mathrm{~nm}$ ) lithographically patterned onto PDMS. The lines have a length of $5 \mathrm{~mm}$ and a width of $150 \mu \mathrm{m}$. Each line has a different orientation angle $\theta$ relative to the longitudinal direction of the straining, $\theta$ varies from $0^{\circ}$ (referred to as the horizontal line) to $90^{\circ}$ (referred to as the vertical line) and relaxed step-see Fig.4(g).

Figure 4 shows optical microscopy images of Au/Ti/SU-8 (50/5/800 nm) orientated lines to the direction of the longitudinal straining direction. Initially, at zero-strain-see Fig. 4(a)-isotropic mechanical process-induced stress led to the formation of micro-bridges on all lines (see Fig. 1Process 1). When the sample is stretched, the micro-bridges gradually disappear as a function of the line orientation and the average interline strain. At $6 \%$ strain, the micro-bridges have 
disappeared for lines with $\theta$ between $90^{\circ}$ and $50^{\circ}$. For these lines, the average longitudinal strain is positive. At $7.8 \%$, cracks appear in lines with $\theta$ between $90^{\circ}$ and $60^{\circ}$ presumably because the uts of the SU-8 is reached. In contrast, two lines now have more micro-bridges due to Poisson effect, beginning at $7.8 \%$ for line with $\theta=10^{\circ}$ and $14.2 \%$ for line with $\theta=0^{\circ}$. The line with $\theta=20^{\circ}$ is special because the results suggest that the Poisson effect and longitudinal strain compensate each other. At a strain equal to $44.7 \%$, if $\theta<20^{\circ}$, there are no cracking in the lines. Interestingly, for the lines between $\theta=30^{\circ}$ and $\theta=60^{\circ}$ at high strains, the cracking is no longer perpendicular to the lines edges. When the applied strain is returned to zero-see Fig. $4(\mathrm{~g})$, the micro-bridges remain in place for low values of $\theta$ and are created again for larger values of $\theta$.

An analysis of the photographs enables a interline strain field to be computed at the various values of applied strain. This is analogous as to what is done automatically and to a much higher resolution in digital image correlation techniques [30]. The evolution of the interline strain field is shown in Figure 5.
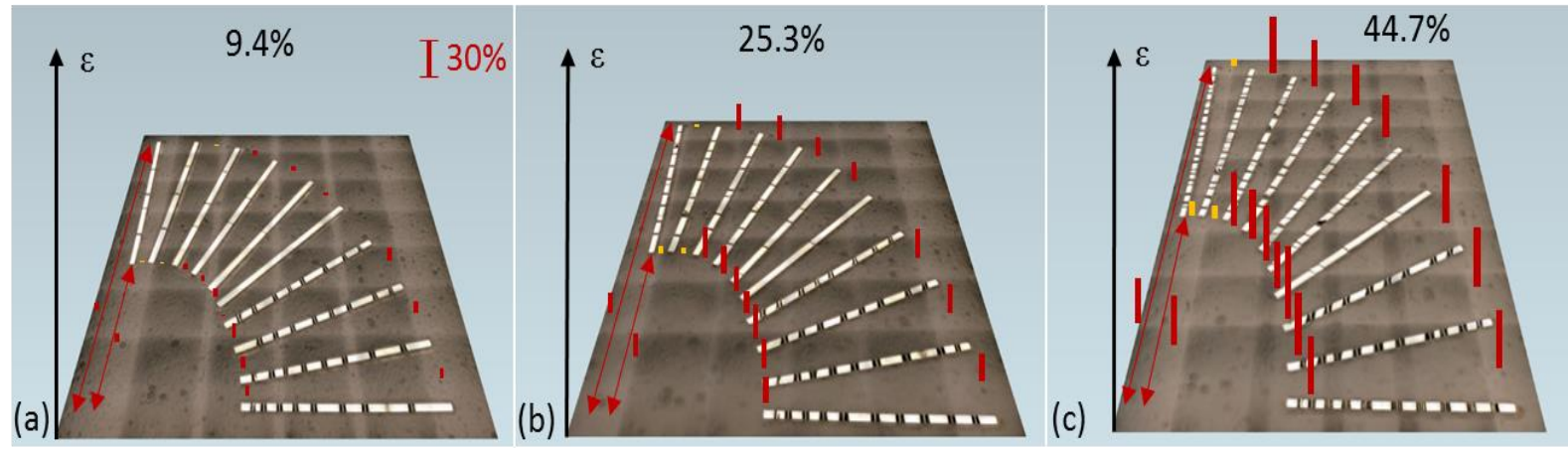

Figure 5. Perspective 3D view of the sample showing the measured interline strain field at average interline strain of (a) $9.4 \%$, (b) $25.3 \%$, and (c) $44.7 \%$. Red lines indicate the interline 
strain in the longitudinal direction and yellow lines indicate interline strain in the transverse direction-due to Poisson effect. The scale bar in (a) corresponds to $30 \%$ strain.
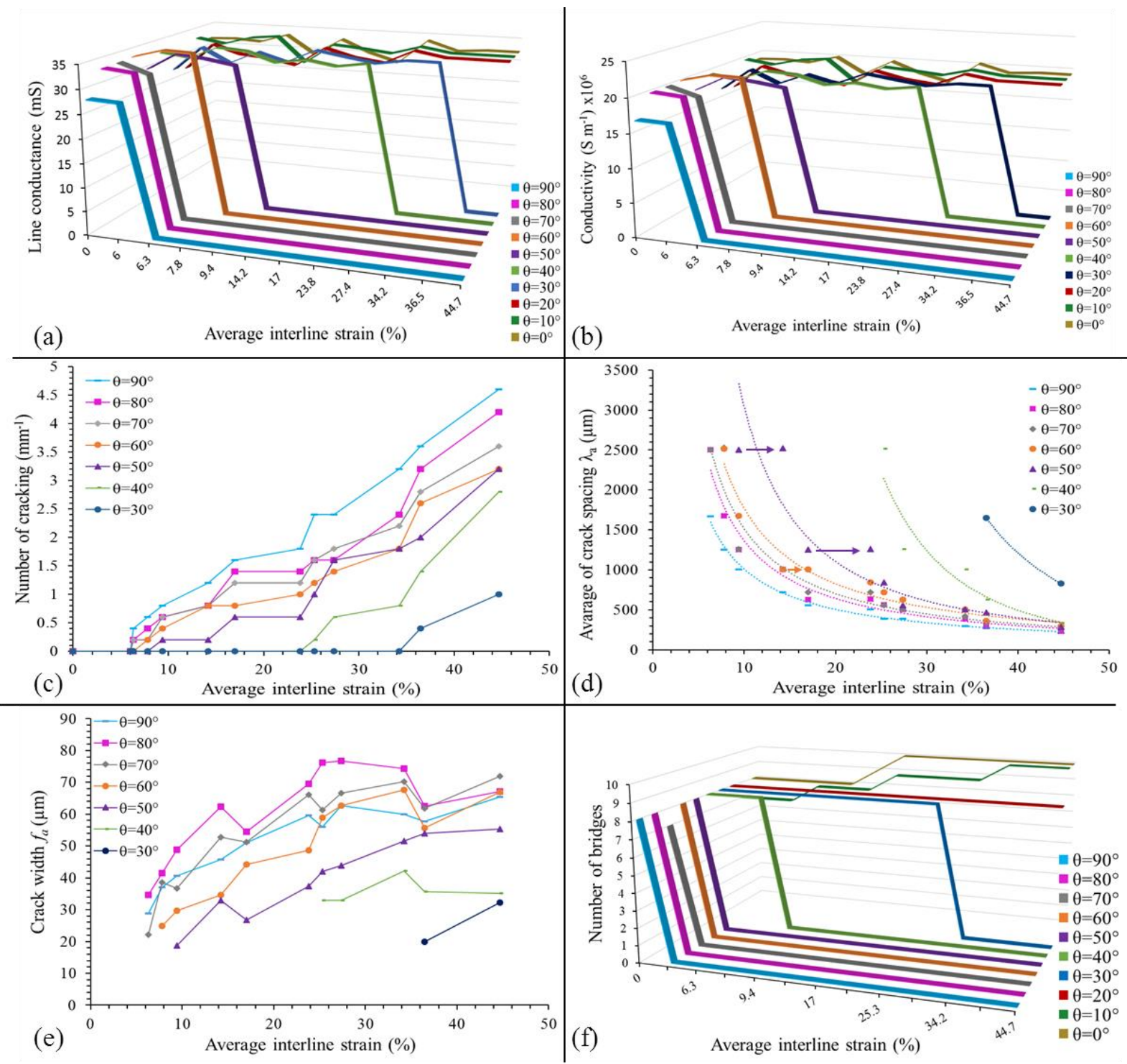

Figure 6. Summary of the electrical characterization, line cracking, and micro-bridge evolution as a function of average interline strain for Au/Ti/SU-8 (50/5/800 nm) lithographically patterned at different orientations compared to the longitudinal external straining. (a) The measured electrical conductance of lines with $\theta$ between $0^{\circ}$ and $90^{\circ}$ as a function of the average interline 
strain. (b) The electrical conductivity of lines with $\theta$ varying between $0^{\circ}$ and $90^{\circ}$ as a function of average interline strain. (c) The number of cracks $\left(\mathrm{mm}^{-1}\right) \mathrm{N}$ of lines with $\theta$ between $30^{\circ}$ and $90^{\circ}$ as a function of average interline strain. (d) The average of crack spacing $\lambda_{a}$ of lines with $\theta$ between $30^{\circ}$ and $90^{\circ}$ as a function of average interline strain. (e) The crack width $f_{a}$ of lines with $\theta$ varying between $30^{\circ}$ and $90^{\circ}$ as a function of average interline strain, and ( $\mathrm{f}$ ) the number of micro-bridges in the lines with $\theta$ varying between $0^{\circ}$ and $90^{\circ}$ as a function of average interline strain.

Figure 6 shows the electrical and mechanical (cracking and micro-bridge evolution) results obtained from the samples. A number of observations can be made from the lines' electrical and mechanical behaviour shown in Figure 6. With reference to Fig. 6(a), the measured electrical resistance is low and very stable $(32.1 \Omega \pm 2.3 \Omega)$ for all conducting (noncracked) lines. Above an average interline strain of $46.4 \%$, only three lines remains conducting (crack-free) but their electrical resistance remains low and stable. The critical angle $\theta_{c}$ to ensure electrical continuity is thus $\sim 20^{\circ}$ for average interline strains between 0 and $50 \%$. With reference to Fig. $6(\mathrm{~b})$, the average of the resistivity of all lines without cracking is $53.6 \pm 3.9 \mathrm{n} \Omega \mathrm{m}$ $\left(\sim 18.7 \times 10^{6} \mathrm{~S} \mathrm{~m}^{-1}\right)$-this is comparable with the observed resistivity values expected from gold films on high quality polished rigid surfaces [44]. The number of cracks increased as a function of average interline strain-see Fig.6(c). For lines with $\theta$ near to $80^{\circ}$, cracks appear quickly at low strain values. By plotting the average crack spacing [20] versus the average interline strain as shown in the Fig. 6(d) one can show that the results of the experimental results follow a wellknown thin film cracking model [48]. However, interestingly we show here that $\theta$ has an influence in the average of crack spacing for a given applied strain-clearly demonstrating that 
architecture has a key role in flexible systems. Note that in Fig. 6(d), when two data points have the same value, it means there were no cracks between two different values of straining - this is indicated by the arrows in Fig. 6(d). Fig. 6(e) shows a plot of the crack width in the Au/Ti/SU-8 lines versus the average interline strain. When external straining is increased, the cracks width in general increased. Interestingly though, the crack width can diminish in successive straining steps-the reason for this is that mechanical strain can be redistributed in the PDMS (i.e. the formation of new cracks). Fig. 6(f) shows a plot of the evolution of the micro-bridges versus average interline strain. At a relatively low strain value equal to $6 \%$, the lines with $\theta$ between $90^{\circ}$ and $50^{\circ}$ no longer contain any micro-bridges. Conversely, for lines having a low value of $\theta$ equal to $0^{\circ}$ and $10^{\circ}$, micro-bridges are formed as the longitudinal strain is increased, presumably due to the Poisson effect. Finally, for line orientated $\theta=20^{\circ}$, no more micro-bridges and no cracking appear during the experiment (up to $46.4 \%$ average interline strain).

\section{Line cracking as the line orientation angle is varied}

We have developed a simple mechanical model (see Supplementary Information) that captures the main observations discussed in the previous section. These observations are: (i) lines at low angles $\theta \leq 20^{\circ}$ undergo a compressive strain and do not break-even at high strain, (ii) lines for which $\theta \geq 30^{\circ}$ incur progressive cracking as the strain is increased, and (iii) lines orientated at higher angles crack at low values of strain.

Our model is a linear spring-based model that calculates the strain experienced by the metallized SU-8 lines at different line orientation angles and for varying applied strain applied to 
the rectangular PDMS block-onto which the lines are fabricated. The model is a function of the geometrical dimensions, the Poisson coefficient of the PDMS, and the Young modulus of PDMS, SU-8, Ti and Au; all these quantities are well known and no fitting or adjusting parameters are necessary. Although the model qualitatively captures the essentials of our experiment, the breaking of the lines is a non-linear phenomenon, as such it should be considered as semiquantitative.

We will now discuss the mechanical strain experienced by the metallized SU-8 layer, as the breaking of the lines occurs when the stress of the SU-8 layer reaches the uts value. As it can be seen in Figure 4, the cracking of the lines is perpendicular to the line's longitudinal axis; hence we focus on the longitudinal component of the SU-8 stress. This longitudinal stress can be easily calculated with our spring-based model (see Supplementary Information for details). In Figure 7 we show the contour plot of the in-line stress in the SU-8 versus the applied strain to the PDMS block (x-axis) and line orientation angle $\theta$ (y-axis). The in-line stress is defined in the Supplementary Information. The blue dots in Fig. 7 indicate the experimental conditions ( $\theta$ and strain) at which the practical lines cracked. 


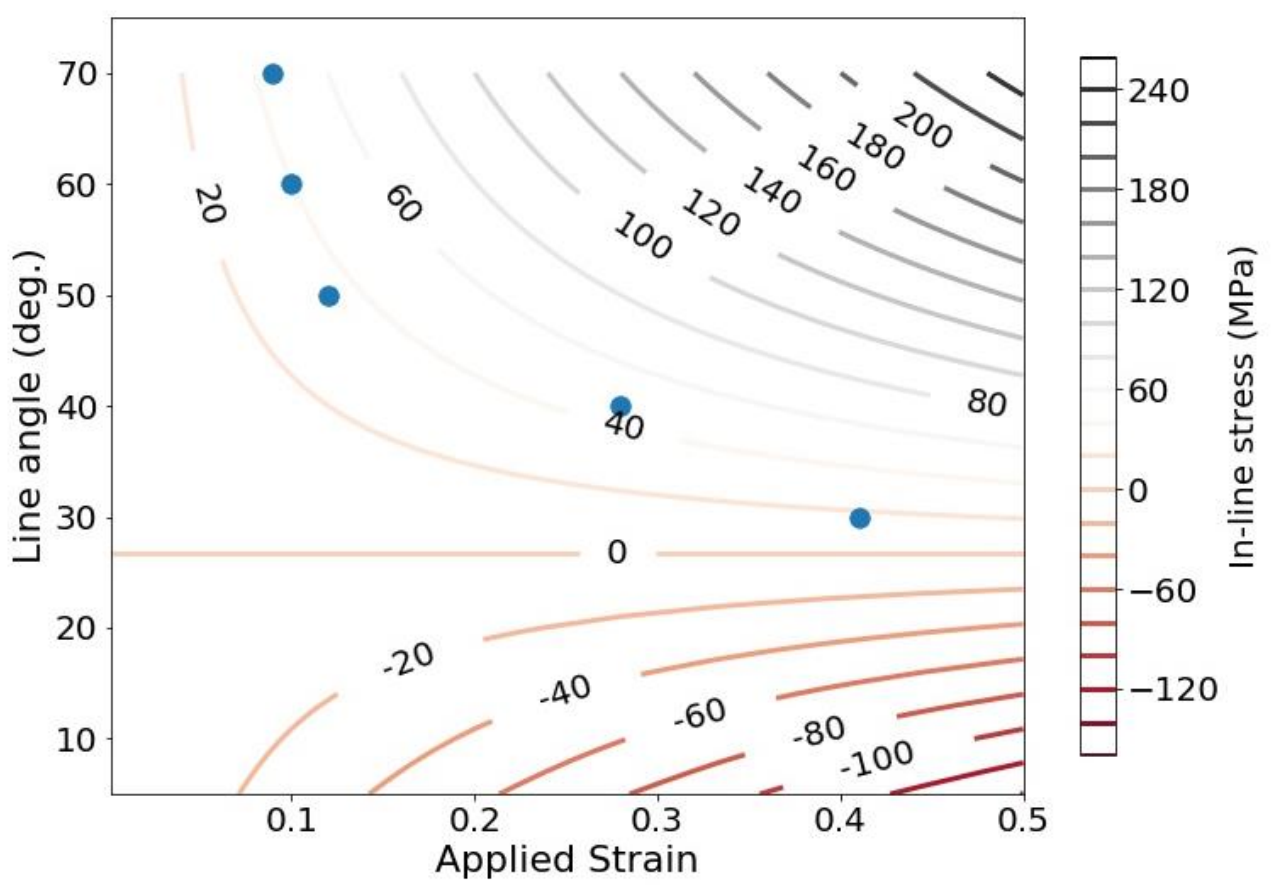

Figure 7. A contour plot of the in-line stress in the metallized SU-8 line as a function of line orientation $\theta$ and applied strain to the PDMS block. The blue dots indicate the experimental conditions ( $\theta$ and strain) when cracking appears in the lines.

Lines orientated at angles lower than 20 degrees did not break. Two zones can be identified in the plot. For $\theta<26^{\circ}$, the longitudinal strain is negative (compressive strain zone), whilst for $\theta>26^{\circ}$ the strain is positive (tensile strain zone). At $\theta=26^{\circ}$ the longitudinal strain is zero, due to perfect compensation of the applied strain and the Poisson effect. These findings are in excellent agreement with our experimental results, namely that lines at angles lower than 30 degrees do not break and experience compressive strain. From a quantitative point of view, we remark that the observed cracking appears at tensile strength on the order of $20 \mathrm{MPa}$. This value is consistent with the range of values of tensile strength of SU-8 found in the literature. 
The experiments and modelling suggest that the Poisson effect leads to compressive straintraversal to the direction of applied strain. The effect of this compressive strain is to reduce the stress in the line-for a given applied strain-as the line orientation angle $\theta$ is reduced. In principle, the transversal Poisson-associated compressive strain cancels the tensile strain along a line having an angle $\theta \approx \tan ^{-1} v_{P D M S}=26.5^{\circ}$ (see Supplementary Information).

\section{Conclusions}

We conclude that innovative process engineering can be used to fabricate electromechanically robust metallized lines and pads onto stretchable PDMS samples. This is achievable by using a patterned SU-8 thin film to shield the metallic thin films from microscopic and macroscopic process-induced and strain-induced cracking which can lead to the electrical resistance of such lines increasing rapidly. Two processes have been successfully developed which enable the fabrication of metallized SU-8 lithographically patterned features directly on PDMS. For example, in the present study transversally-orientated Au/Ti/SU-8 (50/5/800 nm) lineslithographically-patterned on PDMS-have a resistivity of $\sim 60 \mathrm{n} \Omega \mathrm{m}\left(\sim 16.7 \times 10^{6} \mathrm{~S} \mathrm{~m}^{-1}\right)$ at an interline strain of $\sim 40 \%$-this resistivity is comparable to that of both evaporated and pure bulk gold. We have shown that large-area pads can be fabricated-and we have shown the limits of their straining. We have also shown the effect of line orientation relative to the direction of longitudinal straining-the latter experiment has revealed a critical line angle and the importance of architecture on cracking in soft technologies. A simple mechanical modelling agrees well with the study's experimental findings-successfully predicting the appearance of 
the first crack which leads to electrical failure. Our microfabrication processes are compatible with other cleanroom processes and extendible to a full thin film approach. We conclude by saying that although the photoresist SU-8 has been successfully used here as strain shielding element as a proof-of-concept, we note that other photo-patternable materials could no doubt be envisaged for strain-shielding elements for high strain stretchable systems.

\section{Acknowledgements}

The authors would very much like to thank Annie Fattorini and Marc Dewitte for help with the metallization, Christophe Boyaval for help with the SEM, and Flavie Braud for help in fabricating the physical masks using a Tangerine laser machining system (Amplitude Systèmes, France) purchased within the EQUIPEX 'LEAF' project (ANR-11-EQPX-0025). The digital microscope was purchased within the 'TIPTOP_1' project (ANR-16-CE09-0029). This work was partially supported by the French RENATECH network.

\section{References}

[1] Hammock M L, Chortos A, Tee B C-K, Tok J B-H and Bao Z 2013 25th Anniversary Article: The Evolution of Electronic Skin (E-Skin): A Brief History, Design Considerations, and Recent Progress Adv. Mater. 25 5997-6038

[2] Amjadi M, Kyung K-U, Park I and Sitti M 2016 Stretchable, Skin-Mountable, and Wearable Strain Sensors and Their Potential Applications: A Review Adv. Funct. Mater. 26 1678-98

[3] Akinwande D, Petrone N and Hone J 2014 Two-dimensional flexible nanoelectronics Nat. Commun. 5 5678-12 
[4] Kim D-H, Ahn J-H, Choi W M, Kim H-S, Kim T-H, Song J, Huang Y Y, Liu Z, Lu C and Rogers J A 2008 Stretchable and Foldable Silicon Integrated Circuits Science 320 507-11

[5] Heikenfeld J, Drzaic P, Yeo J S and Koch T 2011 A critical review of the present and future prospects for electronic paper J. Soc. Inf. Disp. 19 129-156

[6] White M S, Kaltenbrunner M, Głowacki E D, Gutnichenko K, Kettlgruber G, Graz I, Aazou S, Ulbricht C, Egbe D A M, Miron M C, Major Z, Scharber M C, Sekitani T, Someya T, Bauer S and Sariciftci N S 2013 Ultrathin, highly flexible and stretchable PLEDs Nat. Photonics 7 811-6

[7] Kubo M, Li X, Kim C, Hashimoto M, Wiley B J, Ham D and Whitesides G M 2010 Stretchable Microfluidic Radiofrequency Antennas Adv. Mater. 22 2749-52

[8] Hu L, Pasta M, Mantia F L, Cui L, Jeong S, Deshazer H D, Choi J W, Han S M and Cui Y 2010 Stretchable, Porous, and Conductive Energy Textiles Nano Lett. 10 708-14

[9] Stoppa M and Chiolerio A 2014 Wearable Electronics and Smart Textiles: A Critical Review Sensors 14 11957-92

[10] Folch A 2013 Introduction to BioMEMS (Boca Raton, Fla.: CRC Press)

[11] Woeppel K, Yang Q and Cui X T 2017 Recent advances in neural electrode-tissue interfaces Curr. Opin. Biomed. Eng. 4 21-31

[12] Majidi C 2014 Soft Robotics: A Perspective-Current Trends and Prospects for the Future Soft Robot. 1 5-11

[13] Khang D-Y 2006 A Stretchable Form of Single-Crystal Silicon for High-Performance Electronics on Rubber Substrates Science 311 208-12

[14] Kim K S, Zhao Y, Jang H, Lee S Y, Kim J M, Kim K S, Ahn J-H, Kim P, Choi J-Y and Hong B H 2009 Large-scale pattern growth of graphene films for stretchable transparent electrodes Nature 457 706-10

[15] Rogers J A, Someya T and Huang Y 2010 Materials and Mechanics for Stretchable Electronics Science 327 1603-7

[16] Linghu C, Zhang S, Wang C and Song J 2018 Transfer printing techniques for flexible and stretchable inorganic electronics Npj Flex. Electron. 226

[17] Seghir R and Arscott S 2015 Mechanically robust, electrically stable metal arrays on plasma-oxidized polydimethylsiloxane for stretchable technologies J. Appl. Phys. 118 045309-8 
[18] Lacour S P, Wagner S, Huang Z and Suo Z 2003 Stretchable gold conductors on elastomeric substrates Appl. Phys. Lett. 82 2404-6

[19] Seghir R and Arscott S 2015 Controlled mud-crack patterning and self-organized cracking of polydimethylsiloxane elastomer surfaces Sci. Rep. 5 14787-16

[20] Baëtens T, Pallecchi E, Thomy V and Arscott S 2018 Cracking effects in squashable and stretchable thin metal films on PDMS for flexible microsystems and electronics Sci. Rep. 8 9492-17

[21] Viventi J, Kim D-H, Vigeland L, Frechette E S, Blanco J A, Kim Y-S, Avrin A E, Tiruvadi V R, Hwang S-W, Vanleer A C, Wulsin D F, Davis K, Gelber C E, Palmer L, Van der Spiegel J, Wu J, Xiao J, Huang Y, Contreras D, Rogers J A and Litt B 2011 Flexible, foldable, actively multiplexed, high-density electrode array for mapping brain activity in vivo Nat. Neurosci. 14 1599-605

[22] Someya T, Sekitani T, Iba S, Kato Y, Kawaguchi H and Sakurai T 2004 A large-area, flexible pressure sensor matrix with organic field-effect transistors for artificial skin applications Proc. Natl. Acad. Sci. 101 9966-70

[23] Graz I M, Cotton D P J, Robinson A and Lacour S P 2011 Silicone substrate with in situ strain relief for stretchable thin-film transistors Appl. Phys. Lett. 98124101

[24] Romeo A, Liu Q, Suo Z and Lacour S P 2013 Elastomeric substrates with embedded stiff platforms for stretchable electronics Appl. Phys. Lett. 102 131904-5

[25] Feiner R, Wertheim L, Gazit D, Kalish O, Mishal G, Shapira A and Dvir T 2019 A Stretchable and Flexible Cardiac Tissue-Electronics Hybrid Enabling Multiple Drug Release, Sensing, and Stimulation Small 15 1805526-13

[26] Lorenz H, Despont M, Fahrni N, LaBianca N, Renaud P and Vettiger P 1997 SU-8: a low-cost negative resist for MEMS J Micromech Microeng 7 121-4

[27] Arscott S 2014 SU-8 as a material for lab-on-a-chip-based mass spectrometry Lab. Chip 14 3668-89

[28] Feng R and Farris R J 2003 Influence of processing conditions on the thermal and mechanical properties of SU8 negative photoresist coatings J. Micromechanics Microengineering 13 80-8

[29] Robin C J, Vishnoi A and Jonnalagadda K N 2014 Mechanical Behavior and Anisotropy of Spin-Coated SU-8 Thin Films for MEMS J. Microelectromechanical Syst. 23 168-80

[30] Roland T, Arscott S, Sabatier L, Buchaillot L and Charkaluk E 2011 Digital image correlation of metal nanofilms on SU -8 for flexible electronics and MEMS J. Micromechanics Microengineering 21 125005-8 
[31] Patel J N, Kaminska B, Gray B L and Gates B D 2008 PDMS as a sacrificial substrate for SU8-based biomedical and microfluidic applications J. Micromechanics Microengineering 18 095028-11

[32] Patel J N, Kaminska B, Gray B L and Gates B D 2009 A sacrificial SU-8 mask for direct metallization on PDMS J. Micromechanics Microengineering 19 115014-10

[33] Patel J N, Gray B L, Kaminska B, Wu N-C and Gates B D 2013 SU-8- and PDMS-based hybrid fabrication technology for combination of permanently bonded flexible and rigid features on a single device J. Micromechanics Microengineering 23 065029-10

[34] Jo B-H, Van Lerberghe L M, Motsegood K M and Beebe D J 2000 Three-dimensional microchannel fabrication in polydimethylsiloxane (PDMS) elastomer J. Microelectromechanical Syst. 9 76-81

[35] Wu M-H, Cai H, Xu X, Urban J P G, Cui Z-F and Cui Z 2005 A SU-8/PDMS Hybrid Microfluidic Device with Integrated Optical Fibers for Online Monitoring of Lactate Biomed. Microdevices 7 323-9

[36] Chao T-Y, Hsu M C, Lin C-D and Cheng Y T 2011 SU-8 serial MEMS switch for flexible RF applications J. Micromechanics Microengineering 21 025010-6

[37] Ren $\mathrm{Y}$, Huang S-H, Mosser S, Heuschkel M, Bertsch A, Fraering P, Chen J-J and Renaud P 2015 A Simple and Reliable PDMS and SU-8 Irreversible Bonding Method and Its Application on a Microfluidic-MEA Device for Neuroscience Research Micromachines 6 1923-34

[38] Seghir R and Arscott S 2015 Photo-hardenable and patternable PDMS/SU-8 hybrid functional material: A smart substrate for flexible systems J. Polym. Sci. Part B Polym. Phys. 53 1281-91

[39] Dellmann L, Roth S, Beuret C, Racine G-A, Lorenz H, Despont M, Renaud P, Vettiger P and de Rooij N F 1998 Fabrication process of high aspect ratio elastic and SU-8 structures for piezoelectric motor applications Sens. Actuators Phys. 70 42-7

[40] Mcaleavey A, Coles G, Edwards R L and Sharpe W N 1998 Mechanical properties of SU-8 MRS Proceedings vol 546

[41] Seghir R and Arscott S 2015 Extended PDMS stiffness range for flexible systems Sens. Actuat A 230 33-9

[42] Neugebauer C A 1960 Tensile Properties of Thin, Evaporated Gold Films J. Appl. Phys. 31 1096-101 
[43] Nordström M, Johansson A, Noguerón E S, Clausen B, Calleja M and Boisen A 2005 Investigation of the bond strength between the photo-sensitive polymer SU-8 and gold Microelectron. Eng. 78-79 152-7

[44] Sambles J R, Elsom K C and Jarvis D J 1982 The Electrical Resistivity of Gold Films Philos. Trans. R. Soc. Math. Phys. Eng. Sci. 304 365-96

[45] M. Gaudet and S. Arscott 2017 A user-friendly guide to the optimum ultraviolet photolithographic exposure and greyscale dose of SU-8 photoresist on common MEMS, microsystems, and microelectronics coatings and materials Anal Methods 9 2495-504

[46] Keller S, Blagoi G, Lillemose M, Haefliger D and Boisen A 2008 Processing of thin SU-8 films J. Micromechanics Microengineering 18 125020-10

[47] Adrega T and Lacour S P 2010 Stretchable gold conductors embedded in PDMS and patterned by photolithography: fabrication and electromechanical characterization $J$. Micromechanics Microengineering 20055025

[48] Thouless M D 1990 Crack Spacing in Brittle Films on Elastic Substrates J. Am. Ceram. Soc. $732144-6$ 